\title{
ANÁLISE DAS CARACTERÍSTICAS DE LEGIBILIDADE DA PRESCRIÇÃO DE MEDICAMENTOS E O PERFIL FARMACOTERAPÊUTICO DE PACIENTES HIPERTENSOS
}

\author{
ANALYSIS OF CHARACTERISTICS OF LEGIBILITY OF PRESCRIPTION FOR MEDICATIONS AND THE \\ PHARMACOTHERAPEUTIC PROFILE OF HYPERTENSIVE PATIENTS
}

Vanessa Zandoná Mendes ${ }^{\mathrm{a}^{*}}$, Cicera Cristina Vidal Aragã $\mathbf{b}^{\mathrm{b}^{*}}$, Wilson Sabino $\mathrm{c}^{\mathrm{c}^{* *}}$

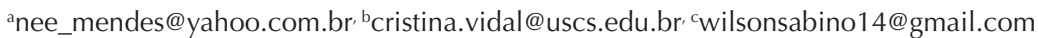

*Universidade Municipal de São Caetano do Sul

"Universidade Federal do Oeste do Pará, Santarém (PA), Brasil

Data de entrada do artigo: 11/02/2014

Data de aceite do artigo: 21/10/2014

\section{RESUMO}

Introdução: Há circunstâncias no processo da prescrição do medicamento, principalmente do paciente hipertenso, que poderiam ser prevenidas e ainda diminuir gastos, como, por exemplo, uma melhor comunicação entre os profissionais médicos, pois esta certamente minimizaria a polimedicação. Objetivo: Analisar as características de legibilidade da prescrição de medicamentos e o perfil farmacoterapêutico de pacientes hipertensos. Método: Trata-se de estudo descritivo com 422 receitas, apresentando nestas, como critério, medicamento anti-hipertensivo dispensado na farmácia-Eescola da Universidade Municipal de São Caetano do Sul no período de agosto de 2012 a dezembro do mesmo ano. Verificouse a legibilidade, classificando as prescriçôes em: legível, pouco legível e ilegível. Foram avaliados os indicadores do uso dos medicamentos recomendados pela Organização Mundial da Saúde. Observando o perfil farmacoterapêutico, os medicamentos foram classificados pelo Índice de Classificação Anatômico Químico e Terapêutico. Resultados: Observou-se que 37,9\% das prescriçôes foram consideradas ilegíveis, por apresentarem um maior grau de dificuldade de leitura e compreensão. Foram prescritos 1.908 medicamentos, encontrando-se 4,5 por prescrição. Deste total, $88 \%$ encontram-se na denominação genérica e $71,5 \%$ estáo padronizados pela Relação Nacional de Medicamentos. Foi observado que $63 \%$ dos medicamentos receitados atuam sobre o sistema cardiovascular, seguido do trato alimentar e metabolismo $(19,6 \%)$, sangue e órgãos hepatopoéticos $(4,9 \%)$ e medicamentos com ação sobre o sistema nervoso $(4,2 \%)$. Conclusáo: Os resultados deste estudo indicam como principais características das prescriçóes analisadas a polimedicaçấo e pouca legibilidade. Quanto ao perfil farmacoterapêutico das receitas dos hipertensos observou-se grande importância na presença de medicamentos inibidores da bomba de prótons, diabetes e os antitrombóticos.

Palavras-chave: Polimedicação; legibilidade; hipertensão; inibidores da bomba de prótons.

\section{ABSTRACT}

Introduction: There are circumstances in the process of prescribing medication, especially with the hypertense patients, which could be avoided and reduce costs, for example: a better communication between the doctors, because that would minimize polymedication. Objective: Analyze the characteristics of the legibility of medication prescription and the pharmacotherapeutic profile of the hypertense patients. Method: This is a descriptive study with 422 recipes, which presented anti-hypertension medication being thrown away by the City University of Sao Caetano do Sul (USCS) pharmacy between August of 2012 and December of the same year. Verified by legibility, classifying the prescriptions as: readable, barely readable, and unreadable. The indicators of the recommended medication that were evaluated are the ones used by 
the World Health Organization. Observing the pharmacotherapeutic profile, the medications were classified by the Anatomic Chemical and Therapeutic index. Results: it was observed that $37.9 \%$ of the prescriptions were unreadable because, when reading the prescriptions, there was a high level of difficulty in reading and comprehension. There were 1,908 medications prescribed, $4.5 \%$ for each prescription. Of this total, $88 \%$ you can find generic dominance and $71.5 \%$ are standardized by the National Medication Relations. 63\% of the medication prescribed acts on the cardiovascular system, followed by the alimentary tract and metabolism (19.6\%), blood and hematopoietic organs (4.9\%) and medications that act on the nervous system (4.2\%). Conclusion: The results in this study indicate prescriptions with polymedication and very little readability as main characteristics. As for the pharmacotherapeutic profile of the hypertensive prescriptions it was observed the importance, of the presence of proton-pump inhibitor in medication, diabetes and the antithrombotics.

Keywords: Polymedication; readability; hypertension; proton-pump inhibitor.

\section{Introdução}

No Brasil ${ }^{1}$, como também em outros países ${ }^{2-5}$, o custo com medicamentos tem um grande impacto econômico nos gastos em saúde que vem apresentando, no Sistema Único de Saúde (SUS), um aumento progressivo no decorrer dos últimos anos ${ }^{6,7}$ - período que sucedeu a implementação da Política Nacional de Medicamentos ${ }^{8}$. Diante deste fato, torna-se possível compreender que uma prescrição realizada sem a devida racionalidade, além de comprometer a saúde da população, pode incrementar ainda mais os gastos deste setor ${ }^{1,6}$.

Segundo a Organização Mundial da Saúde (OMS), mais de 50\% dos medicamentos são prescritos, dispensados ou vendidos de maneira inapropriada'. Metade dos pacientes não utiliza o medicamento corretamente, ou seja, o uso não é racional. Para que seja utilizado de maneira adequada, segundo a OMS, o medicamento precisa ser recebido pelo usuário na dose correta por um período de tempo suficiente, com o menor custo para ele e para a comunidade.

Há, no Brasil, a Lei 5.991/73, apropriada para observância destes indicadores ${ }^{10}$, cabendo ao farmacêutico a responsabilidade de analisar a prescrição em seus aspectos estruturais no momento da dispensação. Além desses aspectos, observa-se que,, as prescriçóes devem adotar obrigatoriamente a Denominação Comum Brasileira ${ }^{11}$ no SUS.

$\mathrm{Na}$ finalidade de verificar a utilização dos medicamentos, a OMS ${ }^{12}$ estabeleceu com especialistas os indicadores do uso de medicamentos para que fossem avaliados: média de medicamento por prescrição, prescrição de medicamentos com nome genérico, porcentagem de medicamentos essenciais, porcentagem de antibióticos e porcentagem de injetáveis.

Entre as doenças que mais exigem cuidados na tomada de decisão em relação à observância da característica da prescrição medicamentosa encontra-se a hipertensão arterial, que, em muitos casos, contém esquemas terapêuticos que apresentam geralmente mais que dois medicamentos por receita ${ }^{13}$, caracterizando a polifarmacoterapia ${ }^{14}$.
A polifarmacoterapia traz consigo, além das possíveis interaçôes medicamentosas ${ }^{15,16}$, a probabilidade do uso de medicamentos que são considerados inapropriados $^{17}$ às pessoas em idade mais avançada. De fato, Rothberg ${ }^{18}$ constatou em seu estudo que a cardiologia foi a especialidade que apresentou maior quantidade de medicamentos prescritos de maneira inadequada (48\%) e que a geriatria foi a que apresentou a menor proporção (24\%). Somado a este processo, a falta de clareza na descriçáo medicamentosa por parte dos prescritores pode confundir e aumentar ainda mais os possíveis problemas relacionados à medicação ${ }^{19,20}$ que afetam diretamente a saúde do usuário.

Constatando-se que o acesso de um paciente aos serviços de saúde e aos próprios medicamentos não é suficiente para garantir êxito no tratamento ${ }^{7,21}$, o conhecimento sobre as características da prescrição e sobre o perfil farmacoterapêutico de uma população pode, por sua vez, contribuir para reduzir problemas relacionados ao uso da medicaçáo, maior êxito no tratamento e redução dos custos individuais e públicos.

O município de São Caetano do Sul possui uma populaçáo de 149.263 habitantes, sendo $23,5 \%$ adultos jovens, com idades entre 20 e 34 anos; $35,8 \%$ adultos, com idades entre 35 a 59 anos; e 19,1\% de idosos, com idade acima de 60 anos encontrando-se acima da média brasileira que é de $10,8 \%$.

A farmácia-escola da Universidade Municipal de São Caetano do Sul (Farma-USCS) foi inaugurada no ano de 2003, estruturando-se a partir de uma parceria com a Secretaria de Saúde de São Caetano do Sul, integrandose ao Programa de Assistência Farmacêutica Municipal. Os medicamentos manipulados neste estabelecimento são distribuídos aos usuários atendendo a prescriçôes médicas provenientes das redes pública e privada do município.

Assim, o presente trabalho tem, por objetivo, analisar as características de legibilidade da prescrição de medicamentos e o perfil farmacoterapêutico dos usuários hipertensos atendidos na farmácia-escola da Universidade Municipal de São Caetano do Sul (Farma-USCS). 


\section{Método}

Este é um estudo retrospectivo e descritivo que está avaliando os indicadores de prescrição propostos pela OMS, a partir de receitas dos medicamentos anti-hipertensivos dispensados na Farma-USCS no período de agosto de 2012 a dezembro do mesmo ano.

Este projeto conta com aprovação do Comitê de Ética em Pesquisa da Universidade Municipal de São Caetano do Sul no 255.792.

Para análise do perfil sociodemográfico dos usuários de anti-hipertensivos foram consideradas as variáveis: sexo, idade e diagnóstico médico. Os registros de estudo desses foram obtidos através de uma base de dados existente na Farma-USCS.

Verificou-se a legibilidade, classificando as prescrições em legível, pouco legível e ilegível, sendo aqui adotada a Metodologia de Rosa ${ }^{23}$. As prescriçóes foram avaliadas por alunos do primeiro ano de farmácia, inexperientes em farmácias e drogarias, com objetivo único de que os vícios de leitura fossem isolados.

Foram avaliados os indicadores do uso dos medicamentos recomendados pela Organizaçáo Mundial da Saúde, sendo avaliados a média de medicamentos prescritos por prescriçáo médica, o percentual de medicamentos prescritos com denominaçáo genérica e o percentual de antimicrobianos prescritos. Utilizou-se da Relação Nacional de Medicamentos Essenciais (RENAME) para identificação da proporção de medicamentos padronizados por receita médica.

$\mathrm{Na}$ observância do perfil farmacoterapêutico, os medicamentos apresentados pela nomenclatura constante na Denominação Comum Brasileira (DCB) foram classificados pelo Índice de Classificação Anatômico Químico e Terapêutico (ATC), para o conhecimento das classes terapêuticas mais prescritas.

Foi calculada a frequência simples dos dados através da análise das prescriçóes utilizando uma planilha eletrônica (Excel), em seguida sendo realizada a análise descritiva dos dados.

\section{Resultados}

Dos 422 usuários de medicamentos para hipertensão atendidos na Farma-USCS, a média de idade foi de $67 \pm 11,8$ anos, sendo que $72,6 \%$ apresentaram idade igual ou maior que sessenta anos. Desses, a maior proporção foi de mulheres $(46,2 \%)$.

Observa-se, na Tabela 1, que foram prescritos 1.908 medicamentos, sendo 4,5 por prescrição. Deste total, $88 \%$ encontram-se na denominação genérica e $71,5 \%$ estâo padronizados pela RENAME.
Tabela 1: Indicadores de uso do medicamento recomendado pela Organização Mundial da Saúde (OMS). Prescrições recebidas na Farma-USCS, município de São Caetano do Sul (SP), 2012

\begin{tabular}{lcccc}
\hline Medicamentos & $\begin{array}{c}\text { Número } \\
\text { absoluto }\end{array}$ & $\begin{array}{c}\text { Média } \\
\text { por } \\
\text { prescriçáo }\end{array}$ & Magnitude & $\begin{array}{c}\text { Recomendação } \\
\text { (OMS) }\end{array}$ \\
Prescritos & 1908 & 4,5 & - & $2,0 \%$ \\
$\begin{array}{l}\text { Denominação } \\
\text { genérica }\end{array}$ & 1682 & 4,0 & $88 \%$ & $100 \%$ \\
$\begin{array}{l}\text { Padronizado } \\
\text { *RENAME }\end{array}$ & 1365 & 3,5 & $71,5 \%$ & $70 \%$ \\
$\begin{array}{l}\text { Antibióticos } \\
\text { Injetáveis }\end{array}$ & - & - & - & $20 \%$ ou menos \\
\hline
\end{tabular}

*Relação Nacional de Medicamentos Essenciais (RENAME)

Quanto às prescriçóes, observa-se na Tabela 2 que $37,9 \%$ foram consideradas ilegíveis por apresentarem um maior grau de dificuldade de leitura e compreensão. Neste primeiro momento denota-se na análise que quanto maior a quantidade de medicamentos por prescrição, 5 a 14 medicamentos (22,3\%), mais ilegíveis tornam-se as receitas.

Tabela 2: Frequência quanto à quantidade de medicamentos por prescrição e a grafia encontrada nas prescrições de medicamentos apresentadas na Farma-USCS, no período de agosto/ dezembro. Município de São Caetano do Sul (SP), 2012

\begin{tabular}{ccccccccc}
\hline $\begin{array}{c}\text { Quantidade } \\
\text { de medica- } \\
\text { mento por } \\
\text { prescriçáa }\end{array}$ & Legível & $\begin{array}{c}\text { Legibilidade } \\
\text { Pouco } \\
\text { legível }\end{array}$ & Ilegível & Total \\
1 a 4 & 73 & $17,3 \%$ & 63 & $14,9 \%$ & 66 & $15,6 \%$ & 202 & $47,9 \%$ \\
5 a 9 & 51 & $12,1 \%$ & 61 & $14,5 \%$ & 85 & $20,1 \%$ & 197 & $46,7 \%$ \\
10 a 14 & 4 & $0,9 \%$ & 10 & $2,4 \%$ & 9 & $2,2 \%$ & 23 & $5,5 \%$ \\
Total & 128 & $30,3 \%$ & 134 & $31,8 \%$ & 160 & $37,9 \%$ & 422 & $100 \%$ \\
\hline
\end{tabular}

Fonte: farmácia-escola da Universidade Municipal de Sấo Caetano do Sul. Elaboração própria

Na Tabela 3 são apresentados os grupos de medicamentos mais prescritos aos usuários de medicamentos para hipertensão da Farma-USCS, de acordo com a classificação anatômica (Nível 1) e terapêutica (Nível 2) da ATC.

Em relação à primeira, 63\% dos medicamentos receitados atuam sobre o sistema cardiovascular, seguido pelos que atuam no trato alimentar e metabolismo (19,6\%), sangue e órgãos hematopoiéticos (4,9\%) e medicamentos com ação sobre o sistema nervoso (4,2\%). Considerando a classificação anatomoterapêutica, entre os oito medicamentos mais consumidos, figuram cinco com ação sobre o sistema cardiovascular: agentes 
Tabela 3: Distribuição dos medicamentos utilizados por usuários da Farma-USCS, segundo a classificação anatomoterapêutica (níveis 1 e 2 da Anatomical Therapeutical Chemical Index (ATC)). Município de São Caetano do Sul (SP), 2012

\begin{tabular}{|c|c|c|c|}
\hline Medicamentos & Código ATC & $\mathbf{N}^{\circ}$ abs & $\%$ \\
\hline Sistema cardiovascular & $\mathrm{C}$ & 972 & 63,0 \\
\hline Agentes sobre o sistema renina-angiotensina & $\mathrm{C} 09$ & 436 & 28,2 \\
\hline Diuréticos & $\mathrm{C} 03$ & 199 & 12,9 \\
\hline Betabloqueadores & $\mathrm{C} 07$ & 113 & 7,3 \\
\hline Antilipêmicos & $\mathrm{C} 10$ & 133 & 8,6 \\
\hline Bloqueadores dos canais de cálcio & $\mathrm{C} 08$ & 58 & 3,8 \\
\hline Cardioterápicos & $\mathrm{C} 01$ & 20 & 1,3 \\
\hline Anti-hipertensivos & $\mathrm{C} 02$ & 13 & 0,8 \\
\hline Trato alimentar e metabolismo & A & 303 & 19,6 \\
\hline Antiácidos/Antiulcerosos/Antiflatulentos & A02 & 129 & 8,4 \\
\hline Medicamentos usados no diabetes & A10 & 114 & 7,4 \\
\hline Vitaminas & A11 & 29 & 1,9 \\
\hline Suplementos minerais & A 12 & 30 & 1,9 \\
\hline Sangue e órgãos hematopoiéticos & B & 75 & 4,9 \\
\hline Medicamentos antitrombóticos & B01 & 67 & 4,4 \\
\hline Preparados antianêmicos & B03 & 5 & 0,3 \\
\hline Substitutos do sangue e soluções de perfusão & B05 & 3 & 0,2 \\
\hline Sistema nervoso & $\mathrm{N}$ & 65 & 4,2 \\
\hline Analgésicos & N02 & 63 & 4,1 \\
\hline Psicoanaléptico & N06 & 2 & 0,1 \\
\hline Aparelho locomotor & M & 59 & 3,8 \\
\hline Medicamentos utilizados para o tratamento da gota & M04 & 33 & 2,1 \\
\hline Biofosfanatos & M05 & 25 & 1,6 \\
\hline Anti-inflamatórios e antirreumáticos não esteróides & M01 & 1 & 0,1 \\
\hline Preparados hormonais sistêmicos, exceto hormônios sexuais & $\mathrm{H}$ & 57 & 3,7 \\
\hline Hormônios da tireoide e antitireoidiano & $\mathrm{H} 03$ & 53 & 3,4 \\
\hline Corticosteroide & $\mathrm{H} 02$ & 4 & 0,3 \\
\hline Outros & G-J-L-S-R & 13 & 0,8 \\
\hline Total & & 1544 & 100 \\
\hline
\end{tabular}

Frequências absolutas e relativas calculadas desconsiderando os medicamentos para os quais não foi possível uma classificaçâo $(\mathrm{n}=364)$

inibidores do sistema renina-angiotensina $(28,2 \%)$, diuréticos $(12,9 \%)$, betabloqueadores e bloqueadores dos canais de cálcio $(11,1 \%)$ e antilipidêmicos $(8,6 \%)$. Seguem-se a estes, os antiácidos/antiulcerosos/antiflatulentos $(8,4 \%)$, medicamentos utilizados no diabetes $(7,4 \%)$ e os antitrombóticos $(4,4 \%)$.

\section{Discussão}

Muito se tem discutido sobre o aumento dos gastos em saúde, principalmente impulsionado pelo custo dos medicamentos ${ }^{1,5}$. A Política Nacional de Medicamentos ${ }^{8}$ trouxe como uma das suas principais diretrizes e prioridades a Assistência Farmacêutica, com a responsabilidade do acesso ao medicamento dividido entre os três entes federados. Contudo, após sua implantação, observou-se a preocupação dos gestores municipais em suprir o acesso aos medicamentos essenciais, porém esquecendo-se ou deixando de lado a racionalidade no uso destes ${ }^{1}$. Possivelmente o reflexo desta prática somado a outras variáveis, como as mudanças demográficas, polimedicação não racional, falta de legibilidade das prescriçóes e prováveis práticas sem evidências científicas, podem estar contribuindo para o aumento destes gastos em saúde ${ }^{1,6}$.

A transição demográfica ${ }^{24}$ que ainda está por se completar no país apresenta como um dos principais resultados o envelhecimento populacional, obrigando gestores, e, principalmente, profissionais que prescrevem os medicamentos, a refletir mais sobre o atual contexto, para uma tomada de decisão mais consciente. $\mathrm{O}$ aumento da longevidade da população brasileira principalmente em determinadas regióes deve ser trazido à luz desta 
discussão porque origina em seu seio o surgimento de doenças crônicas não transmissíveis. Nos últimos anos as doenças degenerativas de aparição tardia, como a enfermidade de Alzheimer, já é prevalente em países europeus. ,O município em estudo incrementa muito os gastos em saúde por apresentar $19,1 \%$ de idosos ${ }^{22}$. No âmbito municipal, esta situação eleva muito o consumo de medicamentos por prescrição quando comparado às populaçóes de estudo que não apresentam este contingente $^{25}$. O presente estudo evidencia este fato, porque dentre os hipertensos analisados, a maior proporção é de idosos $(72,6 \%)$, que se apresentam polimedicados.

A princípio, parece existir um aparente consenso de que a polimedicação numa população de idade mais avançada é quase inevitável entre os profissionais prescritores $^{26}$. Entretanto, no momento da dispensação de medicamentos, o que se observa como uma das fortes razóes para a politerapia é a pouca comunicação existente entre as especialidades médicas, pois cada um prescreve sem levar em conta, em muitos casos, outra prescrição que porventura possa existir.

Adicionando-se a este fator, a quantidade de prescriçóes ilegíveis também contribui para aumentar os possíveis problemas advindos da terapia medicamentosa, tendo sido este fato também observado em outros estudos $^{20,27}$. Tratando-se de uma população idosa, em muitos casos hipertensa e polimedicada como observada na Farma-USCS, a não compreensão da descrição medicamentosa pode acarretar em complicaçôes de saúde que elevam tanto os custos mensais da família como os do sistema de saúde, despendendo ainda mais recursos materiais e humanos para solucionar um agravo que poderia ser evitado.

Além desta situação, o que não está sendo observado por muitos profissionais é que o uso contínuo de determinados fármacos pode trazer efeitos adversos no decorrer do tempo, como exemplo a provável hiperuricemia secundária em indivíduos que utilizam tiazídicos. Essa situação não acrescenta mais um medicamento para solucionar a enfermidade, mas também aumenta muito os riscos de interaçôes medicamentosas num público que já tem sua homeostasia corporal alterada devido à idade.

$\mathrm{O}$ uso dos inibidores da bomba de prótons (IBP), como o Omeprazol, é outro exemplo a ser discutido, pois dentre a classe de antiácidos/antiulcerosos, a maior prevalência encontrada nas prescriçóes no presente estudo é deste inibidor $(91,5 \%)$, que provavelmente é usado como preventivo em disfunçôes gástricas causadas pela politerapia. Todavia, essa prática terapêutica necessita de evidências científicas, pois o que conhecemos atualmente pela história natural das doenças é que no adoecimento geralmente há interação de um agente etiológico e um fator de risco. Contudo, quando numa politerapia observamos a prescrição de um IBP para tratar uma "suposta" enfermidade que possa vir a ocorrer, as outras medicaçôes prescritas estão sendo tratadas como agente etiológico ou fator de risco? Qualquer que seja a direção da resposta parece que necessitamos de evidência plausível centrada na prevenção do cuidado ao usuário do medicamento.

\section{Conclusão}

Muitos são os fatores que podem elevar o custo em saúde, além do aspecto da mudança demográfica constatada no país. Há circunstâncias no processo do uso do medicamento que podem prevenir e proteger a saúde da populaçáo e ainda diminuir gastos, como, uma melhor comunicação entre os profissionais, que poderia minimizar muito a polimedicação.

A informatização é parte do nosso cotidiano, e muitos erros motivados pela ilegibilidade podem ser minimizados com uma prescrição legível utilizando esse sistema. Além disso, a mesma ferramenta traz a possibilidade de informação através de evidências plausíveis, que melhoraria muito a tomada de decisão. Atitudes simples, que podem contribuir com uma melhor qualidade de vida para a comunidade e, ainda reduzir custos.

Os resultados deste estudo indicam como principais características prescriçóes com pouca legibilidade e polimedicação. Quanto ao perfil farmacoterapêutico das receitas dos hipertensos, observou-se grande importância na presença de medicamentos antiácidos, sendo a maior parte de inibidores da bomba de prótons, diabetes e os antitrombóticos.

\section{Referências}

1. Vieira FS, Mendes ACR. Evolução dos gastos do Ministério da Saúde com medicamentos. Área de Economia da Saúde e Desenvolvimento Núcleo Nacional de Economia da Saúde, Ministério da Saúde, 2007.

2. Instituto Nacional de la Salud. Memoria 2000. Madrid:Closas-Orcoyen, S.L.; 2001.

3. Buske L. Health care spending rises $4.6 \%$ in 2003. CMAJ. 2004;170(3):325.

4. Moynihan R. Drug spending in North America rose by $11 \%$ in 2003. BMJ. 2004;328:727.

5. Kondro W. Drug spending hits $\$ 24.8$ billion. CMAJ. 2006;175(1):22. Conselho Federal de Farmácia. Código de ética da profissão farmacêutica. Diário Oficial da União 2004.

6. Melo DO, Ribeiro E, Storpirtis S. A importância e a história dos estudos de utilização de medicamentos. Rev Bras Cienc Farm. 2006;42(4):475-85. 
7. Vieira FS. Assistência farmacêutica no sistema público de saúde no Brasil. Rev Panam Salud Pública. 2010;27(2):149-56.

8. Brasil. Ministério da Saúde. Política nacional de medicamentos. DOU. 2001;C(25):23-6.

9. Organização Mundial da Saúde. Medicamentos: uso racional de los medicamentos. Nota descriptiva $n^{\circ} 338$ [Internet]. 2010 [acesso em 30 de Março de 2013]. Disponível em: http://goo.gl/QhxQAq

10. Lei no 5.991 (17 de dezembro de 1973) [acesso em 29 de Março de 2013]. Disponível em: http://www.planalto.gov. br/ccivil_03/leis/L5991.htm

11. Brasil. Agência Nacional de Vigilância Sanitária. Resolução no 16 [Internet]. Diretoria Colegiada; 2007 [acesso em 30 de Março de 2013]. Disponível em: http://s.anvisa.gov.br/ $\mathrm{wps} / \mathrm{s} / \mathrm{r} / \mathrm{pki}$

12. Organização Mundial de Saúde. How to investigate drug use in health facilities: selected drug use indicators. WHO. DAP 93.1; 1993.

13. Galato D, Silva ESD, Tiburcio LDS. Estudo de utilização de medicamentos em idosos residentes em uma cidade do sul de Santa Catarina (Brasil): um olhar sobre a polimedicação. Ciênc Saúde Colet. 2010;15(6):2899-905.

14. Bjerrum L, Sogaard J, Hallas J, Kragstrup J. Polypharmacy in general practice: differences between practitioners. Br J Gen Pract. 1999;49:195-8.

15. Campana ÉMG, Lemos CC, Magalhães MEC, Brandão AA, Brandão AP. Interaçóes e associaçóes medicamentosas no tratamento da hipertensão - bloqueadores alfa-adrenérgicos e vasodilatadores diretos. Rev Bras Hipertens. 2009;16(4):231-6.

16. Moreno AH, Nogueira EP, Perez MPMS, Lima LRO. Atenção farmacêutica na prevenção de interaçóes medicamentosas em hipertensos. Rev Inst Ciênc Saúde. 2007;25(4):373-7.

17. Bueno CS, Bandeira VAC, Oliveira KR, Colet CF. Perfil de uso de medicamentos por idosos assistidos pelo Programa de Atenção ao Idoso (P.A.I.) da UNIJUÍ. Rev Bras Geriatr Gerontol. 2012;15(1):51-61.
18. Rothberg MB, Pekow PS, Liu F, Korc-Grodzicki B, Brennan MJ, Bellantorio S, Heelon M, Lindenauer PK. Potentially inappropriate medication use in hopitalized elders. J Hosp Med. 2008;3(2):91-102.

19. Júnior DPL, Prado MCTA, Abriata JP, Pelá IR. Receta médicas como causante de riesgo de problema relacionados con medicamentos. Seguim Farmacoter 2004,2(2):86-96.

20. Araújo PTB, Uchôa SAC. Avaliação da qualidade da prescrição de medicamentos de um hospital de ensino. Ciênc Saúde Coletiva [periódico na Internet]. 2011 [acesso em 1 de Abril de]; 16(1):1107-14.

21. Oliveira LCF, Assis MMA, Barboni AR. Assistência farmacêutica no sistema único de saúde: da política nacional de medicamentos à atençáo básica à saúde. Ciênc. saúde coletiva [periódico na internet] 2010 [acesso em 18 maio 2012]; 15(3):3561-7.

22. Instituto Brasileiro de Geografia e Estatística. Censo Demográfico 2010. 2011.

23. Rosa MB, Neiva HM, Anacleto TA, Mendes DP, Freitas FO, Lage JB, Perini E. Legibilidade de prescriçóes médicas com medicamentos potencialmente perigosos em um hospital de Belo Horizonte, MG. Rev SBRAFH. 2003;(2):22-8.

24. Frenk J, Frejka T, Bombadilla JL, Stern C, Lozano R, Sepúlveda J, et al. La transición epidemiológica en América Latina. Boletín Oficina Sanit Panam. 1991;111(6):485-96.

25. Santos V, Nitrini SMOO. Indicadores do uso de medicamentos prescritos e de assistência ao paciente de serviços de saúde. Rev Saúde Públ. 2004;38(6)819-26.

26. Silva AL, Ribeiro AQ, Klein $\mathrm{CH}$, Acurcio FA. Utilização de medicamentos por idosos brasileiros, de acordo com a faixa etária: um inquérito postal. Cad Saúde Públ [periodico na internet]. 2012 [acesso em 27 de junho de 2013]; 28(6):1033-1045.

27. Silvério MS, Leite ICG. Qualidade das prescriçôes em Município de Minas Gerais: uma abordagem farmacoepidemiológica. Ver Assoc Med Bras. 2010;56(6):675-80. 